\title{
Endogenous probabilistic reserve sizing and allocation in unit commitment models: cost-effective, reliable and fast
}

\author{
Kenneth Bruninx, Student Member, IEEE, and Erik Delarue, Member, IEEE
}

\begin{abstract}
In power systems with high shares of variable and limitedly predictable renewables, power system operators need to schedule flexible load, generation and storage to maintain the power system balance when forecast errors occur. To ensure a reliable and cost-effective power system operation, novel reserve sizing and allocation methods are needed. Although stochastic formulations of the unit commitment problem allow calculating an optimal trade-off between the cost of scheduling and activating reserves, load shedding and curtailment, these models may become computationally intractable for real-life power systems. Therefore, in this paper, we develop a novel set of probabilistic reserve constraints, which allows internalizing the reserve sizing and allocation problem in a deterministic unit commitment model, considering the full cost of reserve allocation and activation. Extensive numerical simulations show that this novel formulation yields UC schedules that are nearly as costeffective as the theoretical optimal solution of the stochastic model in calculation times similar to that of a deterministic equivalent.
\end{abstract}

Index Terms-Probabilistic reserve constraints, deterministic unit commitment, stochastic unit commitment, wind power, reserve sizing, reserve allocation, reserve activation.

\section{NOMENCLATURE}

$I \quad$ Set of power plants, indexed by $i$.

$J \quad$ Set of time intervals, indexed by $j$.

$K \quad$ Set of energy storage units, indexed by $k$.

$L \quad$ Set of reserve levels, indexed by $l$.

$S \quad$ Set of wind power scenarios, indexed by $s$.

\section{Decision variables}

$\chi_{j, s}$

Curtailment of RES-based generation at time interval $j$ under scenario $s$, MW.

$\chi_{j, l}^{\mathrm{L}+}$

Curtailment of RES-based generation as upward reserve provider at time interval $j$ in reserve level $l$, MW.

$\chi_{j, l}^{\mathrm{L}-} \quad$ Curtailment of RES-based generation as downward reserve provider at time interval $j$ in reserve level $l$, MW.

K. Bruninx and E. Delarue are with the University of Leuven (KU Leuven) Energy Institute, TME Branch (Energy Conversion), Celestijnenlaan 300A box 2421, B-3001 Leuven, Belgium and EnergyVille, Thor Park 8310, B-3600 Genk. K. Bruninx is also with VITO, the Flemish Institute for Technological Research, Boeretang 200, B-2400 Mol.

Corresponding author: Erik Delarue, tel: $+3216 / 322521$, fax: $+3216 / 322985$, e-mail: erik.delarue@mech.kuleuven.be.

Manuscript received November 6, 2015; revised April 6, 2016; revised July $31,2016$.
Load shedding at time interval $j$ under scenario $s$, MW.

Load shedding as upward reserve provider in time interval $j$ in reserve level $l$, MW.

Activation cost of non-spinning reserves provided by fast-starting power plant $i$ at time interval $j$ in reserve level $l, €$.

Activation cost of upward spinning reserves provided by power plant $i$ at time interval $j$ in reserve level $l, €$.

Activation cost of downward spinning reserves provided by power plant $i$ at time interval $j$ in reserve level $l, €$.

$\mathrm{CO}_{2}$-emission cost of conventional power plant $i$ at time interval $j$ under scenario $s, €$.

Fuel cost of conventional power plant $i$ at time interval $j$ under scenario $s, €$.

Ramping cost of conventional power plant $i$ at time interval $j$ under scenario $s, €$.

Start-up cost of conventional power plant $i$ at time interval $j$ under scenario $s, €$.

Output of conventional power plant $i$ at time interval $j$ under scenario $s$, MW.

Net output of energy storage system $k$ at time interval $j$ under scenario $s$, MW.

Non-spinning reserves delivered by power plant $i$ at time interval $j$, MW.

Non-spinning reserves delivered by power plant $i$ at time interval $j$ in reserve level $l$, MW.

Upward spinning reserve provided by power plant $i$ at time interval $j$, MW.

Downward reserve provided by power plant $i$ at time interval $j$, MW.

Upward reserve provided by energy storage system $k$ at time interval $j$, MW.

Downward reserve provided by energy storage system $k$ at time interval $j$, MW.

Upward reserve provided by energy storage system $k$ at time interval $j$ in reserve level $l$, MW. Downward reserve provided by energy storage system $k$ at time interval $j$ in reserve level $l$, MW.

Upward spinning reserve provided by power plant $i$ at time interval $j$ in reserve level $l$, MW. Downward spinning reserve provided by power plant $i$ at time interval $j$ in reserve level $l$, MW. 


\section{Parameters \\ $\lambda^{\phi} \quad$ Value of lost load, $€ / M W h$. \\ $\tau \quad$ Duration of the time interval, h. \\ $P_{s} \quad$ Probability of scenario $s$. \\ $P_{j, l}^{+} \quad$ Probability of activation of reserves scheduled in upward reserve level $l$ at time interval $j$. \\ $P_{j, l}^{-} \quad$ Probability of activation of reserves scheduled in downward reserve level $l$ at time interval $j$. \\ $D_{j} \quad$ Electricity demand at time interval $j$, MW. \\ $D_{j, l}^{+} \quad$ Upward reserve requirement at time interval $j$ in reserve level $l$, MW. \\ $D_{j, l}^{-} \quad$ Downward reserve requirement at time interval $j$ in reserve level $l$, MW. \\ $G_{j, s}^{\mathrm{F}} \quad$ Wind power output at time interval $j$ under sce- nario $s$, MW.}

\section{INTRODUCTION}

$\mathbf{I}$ $\mathrm{N}$ low-carbon power systems with high shares of renewables (RES) ensuring reliability may become an increasingly critical issue. Some forms of RES-based electricity generation, notably wind and solar PV, have a stochastic character, i.e., they are variable (not or only limitedly dispatchable) and to some extent unpredictable. Deviations from what is expected, e.g., forecast errors, need to be overcome with upor downward regulation of dispatchable generation, load or storage. Moreover, the variability of this RES-based generation requires this reserve capacity to be sufficiently flexible. In this regard, novel power system operation methods will be needed to properly size and allocate operational reserves, in order to ensure a reliable and cost-efficient operation of the power system. Optimal reserve scheduling is the result of a tradeoff between (1) the cost of load shedding and curtailment of RES-based generation and (2) the cost of reserve allocation or procurement, i.e., the cost to have reserves available, and the cost of reserve activation or deployment, i.e., the cost of dispatching or activating these reserves [1], [2].

One of these novel power system scheduling methods is the so-called stochastic unit commitment (SUC) model. The direct representation of the uncertainty via a set of scenarios in the unit commitment (UC) model leads to an optimal trade-off between reliability and operational system cost [2]. By imposing a common UC schedule for all scenarios, the sizing of reserves is internalized in a SUC model. Since scheduled units are dispatched in the second-stage dispatch problem, the activation costs of the scheduled reserves are taken into account during the sizing and allocation process, resulting in an optimal trade-off between reliability, curtailment of RES-based generation and the full cost of providing flexibility. In other words, the amount of required reserves is optimized endogenously, a process we will refer to as 'the internalization of the reserve sizing problem'. However, these SUC models are not devoid of disadvantages. First, the computational cost of solving such a SUC problem may be high and may strongly increase with the number of scenarios one considers, depending on the solution technique employed. In addition, solution stability requirements impose a lower limit on the number of scenarios one needs to use to ensure a meaningful solution of the SUC problem [2]. Improved model formulations [3], decomposition techniques and parallelization [4]-[8], advanced scenario reduction methods [9], [10], , relaxations of the problem [11] and hybrid UC formulations with additional, explicit reserve constraints [2] are used to reduce the computational burden of solving SUC problems. Especially scenario-based decomposition and parallelization may strongly reduce the computational effort associated with solving SUC problems, as it breaks, to some extent, the intimate relationship between the number of scenarios and the computational cost [6]-[8]. Second, the quality of the solution of the SUC model is highly dependent on the quality of the scenario generation and reduction techniques used to produce a representative set of scenarios [9], [12]. Third, in real-life power systems, one needs to consider multiple sources of uncertainty and multiple regions, drastically increasing the complexity of the problem at hand. However, if one succeeds in capturing the underlying stochasticity in a sufficiently small set of scenarios, resulting in a tractable SUC problem, this approach yields the optimal decision under uncertainty.

To avoid the computational burden associated with solving a SUC problem and the complex trade-off between the cost of scheduling reserves and reliability during the optimization of the UC schedule, modelers often resort to deterministic unit commitment (DUC) models with explicit, exogenous reserve requirements. Especially probabilistic reserve requirements i.e., reserve sizing based on the probability that a forecast error of a certain size occurs - have gained attention over the last years [13], [14]. For example, Wang et al. [13] show that a probabilistic reserve requirement outperforms other reserve rules in a DUC model when dealing with uncertainty on wind power forecasts. However, most DUC formulations fail to account for the expected cost of activation of these reserves during the allocation process, typically resulting in sub-optimal UC schedules. To improve the performance of DUC formulation, some researchers include a fixed activation cost per reserve provider [15].

Alternative UC formulations, such as robust UC (RUC) models [16], interval unit commitment (IUC) models [17], chance-constrained UC formulations [18] and hybrid methods [2], have been suggested to approximate the performance of the SUC model at a reasonable computational cost. In RUC models potential realizations of wind power generation are represented as an interval around the central forecast, and thus one avoids assumptions regarding individual scenarios [19]. In the IUC model, the set of scenarios used in a SUC problem is reduced to three distinct scenarios: the central forecast, an upper limit scenario and a lower limit scenario [17]. The IUC and RUC formulations are computationally less demanding than the equivalent SUC model because these formulations consider fewer scenarios [17] or an interval around the central forecast [20]. The RUC and IUC model hedge the system against any realization within an interval around the central forecast, thus providing the same level of reliability. Both the RUC and IUC formulation internalize the reserve sizing problem, but do not account for the reserve activation costs during the sizing and allocation process, i.e. while solving the UC problem. The SUC model generally produces less 
conservative, more cost-efficient UC schedules [17], [20]. Chance-constrained UC formulations [18] try to speed up the convergence of a SUC problem or to reduce the conservatism of a DUC formulation by imposing that certain constraints, typically the power balance, should be satisfied with a certain probability. Small and unlikely violations of the constraints are tolerated, effectively relaxing the problem, while guaranteeing a solution with a certain reliability (e.g. an upper limit on the loss of load probability (LOLP)). Similarly, the so-called budget of uncertainty can be used to alleviate the conservatism of the RUC formulation [20]. However, by setting this reliability level or budget of uncertainty ex-ante, the chance-constrained UC and RUC problem no longer guarantee an optimal tradeoff between the cost of providing reserves and the cost of load shedding. Hybrid methods [2] combine a-priori reserve sizing rules and dedicated scenario reduction methods with a SUC formulation in order to speed up the convergence of the SUC problem to a stable solution. Although hybrid methods effectively internalize the reserve sizing problem and account for activation costs during the allocation process, the limited number of scenarios considered may lead to over- or underestimation of the activation probability, thus the expected activation cost, of the scheduled reserves [2], [21].

In this paper, we propose a novel set of probabilistic reserve constraints which allows accounting for the probability and cost of activation of reserves during the allocation process in a DUC formulation, effectively allowing (1) internalizing the reserve sizing problem and (2) optimizing the allocation of reserves. The obtained UC schedules are nearly as costeffective as that obtained from an equivalent SUC model and significantly better than the corresponding DUC schedules. The computational cost remains low compared to an equivalent SUC problem and is in the same order of magnitude as that of a DUC problem. Compared to the scientific literature, the added value of the proposed model, referred to as the DUC-PR formulation in the remainder of this paper, lies in the simultaneous (1) internalization of the reserve sizing problem and (2) consideration of reserve allocation and activation costs in the sizing and allocation process without the need for stochastic programming techniques. The DUC-PR model internalizes the reserve sizing problem in the UC optimization, which allows the consideration of inter-temporal constraints and 'trading' of risk over time. Chance-constrained DUC formulations include similar (discretized) probability distributions to introduce, e.g., LOLP (loss of load probability) constraints in the optimization problem [22], [23]. However, during the reserve scheduling process, the reserve activation costs are typically not considered. Xiao et al. [24] present a DUC-PR model similar to that in Section II-A, but simplify the representation of the power system. Ramping constraints, the minimum stable operating point of power plants providing non-spinning reserves and their start-up cost, as well as energy storage systems and the regulation services they may offer, are neglected by Xiao et al. [24]. In contrast, all these elements are considered in full detail in the DUC-PR formulation presented below. Moreover, the value of scheduling and activating downward flexibility will be explicitly monetized during the reserve scheduling process, an effect which is not considered in [24].
The remainder of this paper is organized as follows. First, we present the models employed in this paper. A SUC and DUC model will be used as a reference. The DUC-PR formulation and the reserve activation probability calculation are discussed extensively. Second, the performance of the models is discussed in Section III. Last, a conclusion is formulated.

\section{Methodology}

Each of the UC models discussed below is designed to obtain the least-cost UC schedule that allows meeting the demand for electrical energy subject to given operational constraints and uncertain wind power production, the only source of uncertainty considered in this paper. Reserves are allocated, implicitly (SUC) or explicitly (DUC-PR \& DUC), in these UC models. To isolate the impact of the different approaches to represent uncertainty in a UC model, the starting point for the SUC, DUC and DUC-PR problems will be the same set of 500 wind power forecast scenarios. These scenarios are generated for each day using a scenario generation technique based on Pinson et al. [21], [25], using the statistical characterization of the wind power forecast error described in [14]. The ability of the aforementioned technique to capture the uncertainty on the wind power forecast in a set of discrete scenarios is extensively discussed in [21]. For the SUC model, we employ a modified probability distance-based scenario reduction technique to select a number of critical scenarios (here: 30) for consideration in the SUC problems [9]. For the DUC and DUC-PR problems, we calculate an empirical probability density function of the possible wind power realizations from this set of scenarios for each time step in the optimization, which serves as an input for the reserve sizing procedure (Section II-B). Differences in performance due to mismatches in information available in the different UC models are thereby avoided.

The resulting UC schedules are evaluated in terms of operational cost, curtailment of the uncertain RES-based generation and load shedding, by running Monte-Carlo economic dispatch (ED) simulations for a new, large set of scenarios (here: 500). These scenarios are generated via the aforementioned scenario generation technique [21], [25], using the original statistical characterization of the uncertainty [14]. The dispatch model is set up as a DUC model and is executed for each scenario individually, without any reserve requirements, with the UC status set to that obtained from the DUC, DUC-PR or SUC model and the wind power forecast replaced by the wind power scenario at hand. If non-spinning reserves are considered, faststarting units may start-up and shut-down during dispatch if they are scheduled during the UC optimization. Note that the set of scenarios considered during dispatch is identical in the evaluation of the SUC, DUC-PR and DUC schedules to ensure comparability of the results.

In all the UC models presented in this paper, the network constraints are omitted, as we did in [26]. This assumption may lead to transmission congestion, especially under high wind penetration levels [27]. However, the Belgian power system, which provides the basis for our case study, has enough (internal) transmission capacity to make the effect of 
congestion essentially negligible [28]. Currently, redispatching to alleviate transmission congestion affects $0.08 \%$ of the yearly electricity production and increases the annual operating cost by approximately $0.3 \%$ (2.9 million EUR per year) [28]. Omitting the transmission constraints does therefore not significantly affect the conclusions of this case study. The omission of transmission constraints however significantly reduces the computational burden associated with solving the UC problems. For example, Papavasiliou et al. [29] report that a transmission-constrained SUC model for a relatively small system with 375 transmission lines requires from several to tens of hours to achieve a reasonably small duality gap, even for a relatively small number of scenarios.

Starting from a SUC formulation, the DUC and DUC-PR models are introduced as simplifications of that SUC model. A full description of these UC models can be found in [21] ${ }^{1}$.

\section{A. UC Models}

In a SUC model, the power plants are scheduled and dispatched in such a way that the overall expected operational cost over the simulated time period and a certain set of scenarios is minimized. These scenarios are discrete realizations of the uncertain variable, in this case wind power. The total operational cost consists of fuel $\operatorname{costs} c_{i, j, s}^{\mathrm{F}}$, start-up $\operatorname{costs} c_{i, j, s}^{\mathrm{SU}}$, ramping costs $c_{i, j, s}^{\mathrm{R}}, \mathrm{CO}_{2}$-emission costs $c_{i, j, s}^{\mathrm{CO}_{2}}$ and the cost of load shedding $\left(\lambda^{\phi} \cdot \phi_{j, s}\right.$, with $\phi_{j, s}$ the load shed and $\lambda^{\phi}$ the value of lost load). The objective function reads

$$
\begin{aligned}
& \min \sum_{j} \sum_{s} P_{s}\left(\sum_{i}\left(c_{i, j, s}^{\mathrm{SU}}+c_{i, j, s}^{\mathrm{F}}+c_{i, j, s}^{\mathrm{CO}_{2}}+c_{i, j, s}^{\mathrm{R}}\right)\right. \\
& \left.\quad+\tau \cdot \lambda^{\phi} \cdot \phi_{j, s}\right)
\end{aligned}
$$

where $P_{s}$ is the probability of a scenario $s$ (set $S$ ). The fuel cost $\left(c_{i, j, s}^{\mathrm{F}}\right)$ and the $\mathrm{CO}_{2}$-emission cost are dependent on the output of the power plant $i$ (set $I$ ) on time step $j$ (set $J$ ) in scenario $s$, the fuel used and the technology. Ramping costs are triggered by changes in output of the power plant. The start-up cost $c_{i, j, s}^{\mathrm{SU}}$ is scenario-dependent, as faststarting power plants can have a different UC status in each scenario if they provide non-spinning reserves. For all other units, the optimization procedure determines a common UC schedule that minimizes the overall expected operational cost. The only first-stage variables in this SUC model are thus the commitment status-variables of the power plants, excluding those that are considered to be 'fast-starting'.

This optimization is subjected to a number of constraints. First, the supply and demand for electricity must be equal at all time steps $j$ in each scenario $s$ :

$\forall j, \forall s: \quad D_{j}-\phi_{j, s}=\sum_{i} g_{i, j, s}+G_{j, s}^{\mathrm{F}}-\chi_{j, s}+\sum_{k} g_{k, j, s}^{\mathrm{ES}}$

The demand $D_{j}$ on each time step $j$ is assumed to be known and fixed. This demand must be met in each scenario by (1) electricity generated from dispatchable power plants $g_{i, j, s} ;(2)$ the uncertain wind power forecast $G_{j, s}^{\mathrm{F}}$, which can be curtailed

${ }^{1}$ In [21], the DUC-PR model is referred to as a "probabilistic UC formulation'. $\left(0 \leq \chi_{j, s} \leq G_{j, s}^{\mathrm{F}}\right)^{2}$; (3) the net injection of power from energy storage (ES) systems $g_{k, j, s}^{\mathrm{ES}}$ and (4) the shedding of load $\phi_{j, s}$. The dispatch over a set of scenarios and the constraint of a common UC status - except for fast-starting units - abolishes the need for explicit reserve requirements [2]. Consequently, the full reserve allocation and activation cost are both taken into account directly during the UC optimization. Second, the power plants have several techno-economical constraints, such as a minimum and maximum output level, minimum up and down times and ramping rates, different per fuel and technology. Last, the ES systems are included in the model. The net output of ES system $k\left(g_{k, j, s}^{\mathrm{ES}}\right)$ can be positive (discharging) or negative (charging) and is constrained to the capacity of the ES system. The energy content of the ES system is limited to a minimum and maximum level. The dispatch over all scenarios ensures the technical feasibility of activating the scheduled ES-based reserves [26].

In a DUC model, one only considers the (most-likely) forecast scenario, which is assumed to occur with a probability of 1 . The objective function of the DUC model expresses the minimization of the operational cost under forecast conditions:

$\min \sum_{j}\left(\sum_{i}\left(c_{i, j}^{\mathrm{SU}}+c_{i, j}^{\mathrm{F}}+c_{i, j}^{\mathrm{CO}_{2}}+c_{i, j}^{\mathrm{R}}\right)+\tau \cdot \lambda^{\phi} \cdot \phi_{j}\right)$

Since the DUC formulation only considers one scenario, the index $s$ has been dropped.

To ensure that real-time mismatches between the forecasted wind power production and the realized wind power production can be compensated, explicit reserve requirements are added to the model:

$$
\begin{aligned}
& \forall j: D_{j}^{+} \leq \sum_{i}\left(r_{i, j}^{+}+n s r_{i, j}^{+}\right)+\sum_{k} r_{k, j}^{\mathrm{ES}+}+\chi_{j} \\
& \forall j: D_{j}^{-} \leq \sum_{i} r_{i, j}^{-}+\sum_{k} r_{k, j}^{\mathrm{ES}-}
\end{aligned}
$$

At each time step $j$, sufficient upward $\left(D_{j}^{+}\right)$and downward $\left(D_{j}^{-}\right)$reserves must be available. The reserves provided by online conventional units $\left(r_{i, j}^{+}\right.$and $\left.r_{i, j}^{-}\right)$are restricted by the minimum and maximum stable operating points and the remaining rampable capacity of the power plants. Fast-starting units that are not committed in the forecast scenario (nonspinning reserves, $n s r_{i, j}^{+}$), scheduled wind power curtailment $\left(\chi_{j}\right)$ and regulation services provided by the ES systems $\left(r_{k, j}^{\mathrm{ES}+}\right)$ may further fulfill the upward reserve requirement ${ }^{3}$. Downward reserves may also be offered by the ES systems $\left(r_{k, j}^{\mathrm{ES}-}\right)$. To ensure the real-time availability of the reserves offered by the ES systems, the ES-based reserves are limited to the scheduled output and the capacity of the ES systems, as described in [26]. The feasibility of deploying the scheduled

\footnotetext{
${ }^{2}$ Note that we assume that excess RES-based generation may be curtailed at no explicit cost. Indeed, the only cost perceived by the system operator is the opportunity cost of not utilizing available RES-based generation. An explicit cost for curtailing RES-based generation can be included in the objective function analogously to the cost of load shedding $\left(\sum_{j} \sum_{s} P_{s} \cdot \tau \cdot \lambda^{\phi} \cdot \phi_{j, s}\right)$.

${ }^{3}$ If a shortage of supply occurs in the forecast scenario, the demand for reserves has to be relaxed before load shedding occurs. The introduction of a slack variable, restricted to positive values and penalized at a high cost (less than $\lambda^{\phi}$ ) in the objective function, allows this. For sake of simplicity, this is not included explicitly in the model description.
} 
ES-based reserves is enforced in a worst-case activation scenario (i.e. activation of all reserves in one direction, upward or downward) [26].

In the DUC model, only the allocation costs of spinning reserves are implicitly considered. Indeed, the optimization problem is constrained by the reserve requirements, which trigger additional operational costs, i.e. the reserve allocation costs. By assumption, non-spinning reserves may be scheduled at no explicit cost. As the reserves are not 'dispatched' during the allocation process, the expected cost of activating these reserves is not accounted for during the UC optimization. The model thus has insufficient information available to make a trade-off between spinning, non-spinning reserves and regulation services offered by the ES systems. In addition, load shedding (upward flexibility) and additional real-time curtailment of RES-based generation (downward flexibility) are not considered explicitly as reserve providers, in contrast to the SUC formulation. Implicitly, one assumes that this tradeoff has been made ex-ante in the reserve sizing problem [1].

The DUC-PR model builds further on the DUC formulation above, but includes the reserve requirements in a novel way and accounts for the activation cost of scheduled reserves. The upward and downward reserve requirements are split in $L$ levels, each of which will correspond to a specific activation probability (upward $P_{j, l}^{+}$or downward $P_{j, l}^{-}$). The calculation of the reserve levels and the corresponding activation probabilities is the subject of Section II-B. Reserve requirements (4)-(5) are replaced by Eq. (6)-(7):

$$
\begin{aligned}
\forall j, \forall l: D_{j, l}^{+}= & \sum_{i}\left(r_{i, j, l}^{\mathrm{L}+}+n s r_{i, j, l}^{\mathrm{L}+}\right)+\sum_{k} r_{k, j, l}^{\mathrm{ES}, \mathrm{L}+} \\
& +\chi_{j, l}^{\mathrm{L}+}+\phi_{j, l}^{\mathrm{L}+} \\
\forall j, \forall l: D_{j, l}^{-}= & \sum_{i} r_{i, j, l}^{\mathrm{L}-}+\chi_{j, l}^{\mathrm{L}-}+\sum_{k} r_{k, j, l}^{\mathrm{ES}, \mathrm{L}-}
\end{aligned}
$$

Additional load shedding $\left(\phi_{j, l}^{\mathrm{L}+} \geq 0\right)$ and curtailment of RESbased generation $\left(\chi_{j, l}^{\mathrm{L}-}, \chi_{j, l}^{\mathrm{L}+} \geq 0\right)$ are explicitly considered as flexibility options. Assuming wind power is the only source of uncertainty, upward RES-based reserves are constrained to the 'scheduled' curtailment, i.e. curtailment of the forecasted wind power:

$\forall j: \sum_{l} \chi_{j, l}^{\mathrm{L}+} \leq \chi_{j} \leq G_{j}^{\mathrm{F}}$

If it is cost-optimal not to schedule downward reserves in reserve level $l$, this is allowed by considering additional curtailment $\chi_{j, l}^{\mathrm{L}-}$ of unexpected increases in RES-based generation. Conventional reserves are constrained to the remaining ramping capacity and minimum and maximum stable generation level of power plant $i$, as in the DUC model. The scheduled reserves are allocated to at least one reserve level $l$ :

$\begin{array}{ll}\forall i, \forall j: & \sum_{l} r_{i, j, l}^{\mathrm{L}+}=r_{i, j}^{+} \\ \forall i, \forall j: & \sum_{l} r_{i, j, l}^{\mathrm{L}-}=r_{i, j}^{-}\end{array}$

$$
\begin{aligned}
& \forall i, \forall j: \quad \sum_{l} n s r_{i, j, l}^{\mathrm{L}+}=n s r_{i, j}^{+} \\
& \forall i, \forall j, \forall l: \quad r_{i, j, l}^{\mathrm{L}+}, r_{i, j, l}^{\mathrm{L}-}, n s r_{i, j, l}^{\mathrm{L}+} \geq 0
\end{aligned}
$$

Similarly, we assign the ES-based reserves, restricted to the capacity and scheduled output of the ES systems [26], to one or more reserve levels $l$ :

$$
\begin{aligned}
& \forall k, \forall j: \quad \sum_{l} r_{k, j, l}^{\mathrm{ES}, \mathrm{L}+}=r_{k, j}^{\mathrm{ES}+} \\
& \forall k, \forall j: \quad \sum_{l} r_{k, j, l}^{\mathrm{ES}, \mathrm{L}-}=r_{k, j}^{\mathrm{ES}-} \\
& \forall k, \forall j, \forall l: \quad r_{k, j, l}^{\mathrm{ES}, \mathrm{L}-}, r_{k, j, l}^{\mathrm{ES}, \mathrm{L}+} \geq 0
\end{aligned}
$$

The cost of activating reserves $\left(a c_{i, j, l}^{\mathrm{R}+}, a c_{i, j, l}^{\mathrm{NSR}+}, a c_{i, j, l}^{\mathrm{R}-}, \tau \cdot \lambda^{\phi}\right.$. $\phi_{j, l}^{+}$) can now be explicitly added to the objective function. In the DUC-PR model, Eq. (1) is replaced by

$$
\begin{aligned}
& \min \sum_{j}\left(\sum_{i}\left(c_{i, j}^{\mathrm{SU}}+c_{i, j}^{\mathrm{F}}+c_{i, j}^{\mathrm{CO}_{2}}+c_{i, j}^{\mathrm{R}}\right)+\tau \cdot \lambda^{\phi} \cdot \phi_{j}\right) \\
& +\sum_{j} \sum_{l}\left(P_{j, l}^{+}\left(\sum_{i}\left(a c_{i, j, l}^{\mathrm{R}+}+a c_{i, j, l}^{\mathrm{NSR}+}\right)+\tau \cdot \lambda^{\phi} \cdot \phi_{j, l}^{\mathrm{L}+}\right)\right. \\
& \left.\quad+P_{j, l}^{-} \sum_{i} a c_{i, j, l}^{\mathrm{R}-}\right)
\end{aligned}
$$

The operational cost under forecast conditions (first line in Eq. (16)) is complemented with the reserve activation costs (second and third line in Eq. (16)). These activation costs are dependent on the probability of activation of the reserve level (upward: $P_{j, l}^{+}$, downward: $P_{j, l}^{-}$) and the operational costs associated with each flexibility option (spinning reserves $a c_{i, j, l}^{\mathrm{R}+}$ and $a c_{i, j, l}^{\mathrm{R}-}$, non-spinning reserves $a c_{i, j, l}^{\mathrm{NSR}+}$ or load-shedding $\tau \cdot \lambda^{\phi} \cdot \phi_{j, l}^{+}$) scheduled to provide the reserves in this level. Spinning reserves result in fuel and $\mathrm{CO}_{2}$-emission costs. The activation cost of non-spinning reserves $\left(a c_{i, j, l}^{\mathrm{NSR}+}\right)$ additionally contains start-up costs. As the ramp-rate at which the reserves are activated is unknown, the associated ramping costs are not considered as reserve activation costs during the reserve allocation process. Activating upward reserves will always result in an operational cost increase $\left(a c_{i, j, l}^{\mathrm{R}+}, a c_{i, j, l}^{\mathrm{NSR}+} \geq 0\right)$. Downward reserves may however trigger cost reductions $\left(a c_{i, j, l}^{\mathrm{R}-} \leq 0\right)$, as fuel is saved if conventional generation is replaced by an unexpected increase in RES-based generation. The activation cost of curtailment (downward flexibility) is assumed to be zero $^{4}$, while the expected volume of additional load shedding (upward flexibility, $P_{j, l}^{+} \cdot \tau \cdot \phi_{j, l}^{\mathrm{L}+}$ ) is penalized at the value of lost load $\lambda^{\phi}$.

\section{B. Reserve sizing and probability of activation}

The probability that the scheduled reserves are activated depends on the forecast of intermittent generation, changing from time step to time step. Assuming we have a full description

\footnotetext{
${ }^{4}$ If curtailment of RES-based generation entails an explicit cost for the system operator, these costs can be included in the objective of the DUC-PR problem (Eq. (16)) analogously to the cost of load shedding under forecast conditions $\left(\sum_{j} \tau \cdot \lambda^{\phi} \cdot \phi_{j}\right)$ and as a reserve provider $\left(\sum_{j} \sum_{l} P_{j, l}^{+} \cdot \tau \cdot \lambda^{\phi}\right.$. $\left.\phi_{j, l}^{\mathrm{L}+}\right)$.
} 


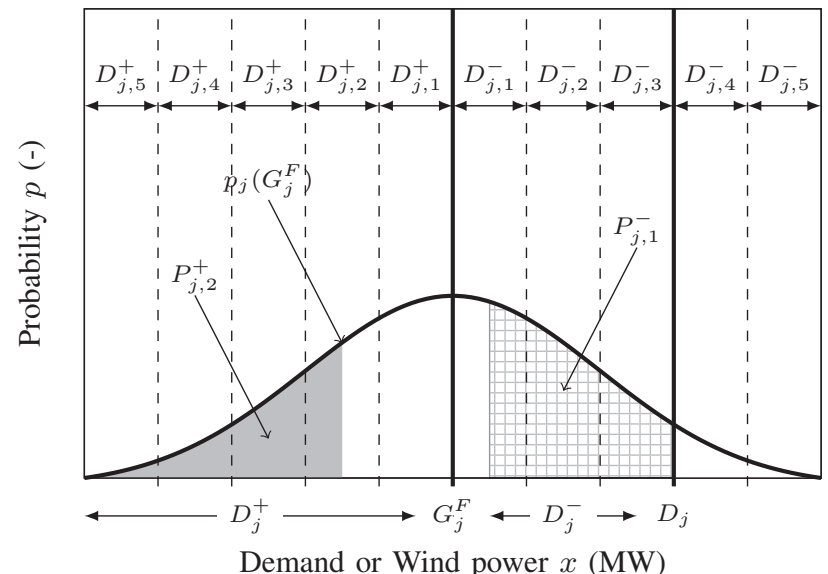

Fig. 1. Assuming that the probability distribution of the forecast error $\left(p_{j}\left(G_{j}^{F}\right)\right)$, the demand $D_{j}$ and the forecasted wind power production $G_{j}^{F}$ are known, one can calculate the probability of a positive or negative forecast error occuring, thus the probability of activation of some predefined reserve levels. For example, with $L=5$, the probability of activation of reserves scheduled in $D_{j, 2}^{+}$is given by the grey area $\left(P_{j, 2}^{+}\right)$. Likewise, the probability of activation of $D_{j, 1}^{-}\left(P_{j, 1}^{-}\right)$is given by the shaded surface. Note that interval on which the activation probability of downward reserves is calculated is limited by the demand $D_{j}$. The form of distribution $p_{j}\left(G_{j}^{F}\right)$ is purely illustrative.

of the probability density function of the wind power forecast error $\left(p_{j}\left(G_{j}^{F}\right)\right)$, the different reserve levels and the associated probability of activation can be calculated as follows. First, we divide the domain of the probability density function $p_{j}\left(G_{j}^{F}\right)$ in a predefined number of intervals $2 L$ ( $L$ intervals for positive forecast errors, $L$ intervals for negative forecast errors) (Fig. 1). Each of the resulting intervals corresponds to a reserve level $\left(D_{j, l}^{+}\right.$and $\left.D_{j, l}^{-}\right)$. Second, the probability of activation of reserves scheduled in each of these intervals can be determined via Eq. (17)-(18), assuming a uniform discretization and with $\Delta D$ the width of the reserve levels:

$\begin{array}{ll}\forall j, \forall l: & P_{j, l}^{+}=\int_{-\infty}^{\mathrm{G}_{\mathrm{j}}^{\mathrm{F}}-\left(1-\frac{1}{2}\right) \cdot \Delta \mathrm{D}} p_{j}\left(G_{j}^{\mathrm{F}}\right) d x \\ \forall j, \forall l: & P_{j, l}^{-}=\int_{\mathrm{G}_{\mathrm{j}}^{\mathrm{F}}+\left(1-\frac{1}{2}\right) \cdot \Delta \mathrm{D}}^{\mathrm{D}_{\mathrm{j}}} p_{j}\left(G_{j}^{\mathrm{F}}\right) d x\end{array}$

In a DUC model, reserves are typically sized to cover a certain percentage of the range of forecast errors [14]. For the case study below, we quantify the upward $D_{j}^{+}$and downward $D_{j}^{-}$reserve requirements at each time step as the smallest quantities that cover the full range of the wind power forecast error at each time step (Fig. $1, D_{j}^{+}$and $D_{j}^{-}$). This makes the total reserve requirement comparable in the DUC and DUCPR simulations, as in this case by definition $\sum_{l} D_{j, l}^{-}=D_{j}^{-}$ and $\sum_{l} D_{j, l}^{+}=D_{j}^{+}$.

The reserve requirements in the DUC and DUC-PR problems are limited to the 'useful' domain: only reserve requirements that could avoid load shedding or curtailment of RESbased generation are considered. For example, the demand for downward reserves is limited to the amount of RES-based generation that can be absorbed by the power system (no downward reserves above $D_{j}$, Fig. 1).

\section{CASE STUDY}

In Section III-A, we explore the impact of the number of reserve levels employed in the DUC-PR problem and compare the scheduled flexibility to that obtained with the SUC and DUC models. In Section III-B and III-C, the resulting operational cost and computational performance of the DUCPR model are compared to that of an equivalent SUC and DUC model, based on simulations for four representative weeks.

The simulations are run for a power system inspired on the Belgian power system, assuming a $30 \%$ wind power penetration (annually, energy basis). The peak demand at transmission system level in this system typically occurs in winter time and equals about $14 \mathrm{GW}$, while the lowest demand - around $6 \mathrm{GW}$ - typically occurs during daytime in the summer. The annual Belgian electricity demand at transmission level amounts to about 83 TWh [30]. Electrical energy generated from RES other than wind (7\% of the annual electric energy demand) is treated as a demand correction and cannot be curtailed. The demand profile and wind power data (2013) are obtained from Elia, the Belgian TSO [30]. The Belgian conventional generation system, consisting of 71 power plants and combined-heat-and-power plants, in total 13,920 MW of dispatchable capacity, has been taken from Elia [30]. The nominal efficiency of the plants is based on the type, the fuel and the age of the power plant [21]. Open-cycle gas turbines and oil-fired units with a size of less than $100 \mathrm{MW}$, a minimum up- and down time of one time step and the capability to ramp from zero output to full capacity within a time step are considered as 'fast-starting units'. In total 35 fast-starting units (with a cumulative capacity of 1,118 MW) are considered in this case study. One pumped hydro ES (PHES) system has been included, with a maximum capacity of 1,308 MW, a round-trip efficiency of $75 \%$ and a storage capacity of 3,924 $\mathrm{MWh}$. The minimum energy content of the storage facility is set to $10 \%$ of its storage capacity. The $\mathrm{CO}_{2}$-price is set to $10 € / \mathrm{t} \mathrm{CO}_{2}$. The value of lost load is set to $10,000 € / \mathrm{MWh}$. Curtailment is assumed to be free.

The planning horizon is 24 hours. The time step equals 15 minutes, in order to adequately capture the impact of the uncertain wind power generation on the scheduling and dispatching of the conventional power plants and energy storage systems, as advocated by Pandžić et al. [17] and Troy et al. [31]. Note that the time resolution of a UC model may have a significant impact on the cost-effectiveness of the resulting UC schedules and the computational cost to obtain those solutions.

The UC models are implemented in GAMS 24.4 and MATLAB 2012b. CPLEX 12.6 is used as solver. Calculations are run on the ThinKing HPC cluster of the KU Leuven, using a $2.8 \mathrm{GHz}$ machine with 20 cores and $64 \mathrm{~GB}$ of RAM. The optimality gap was set to $0.5 \%$.

\section{A. Reserve levels \& scheduled flexibilty}

In this section, we study the behavior of the DUC-PR model in detail based on simulations performed for one particular day (the first day of week 39). On this day, the forecasted wind power ramps up from $1,000 \mathrm{MW}$ to $6,000 \mathrm{MW}$, capable of covering approx. $47 \%$ of the demand for electrical energy. 


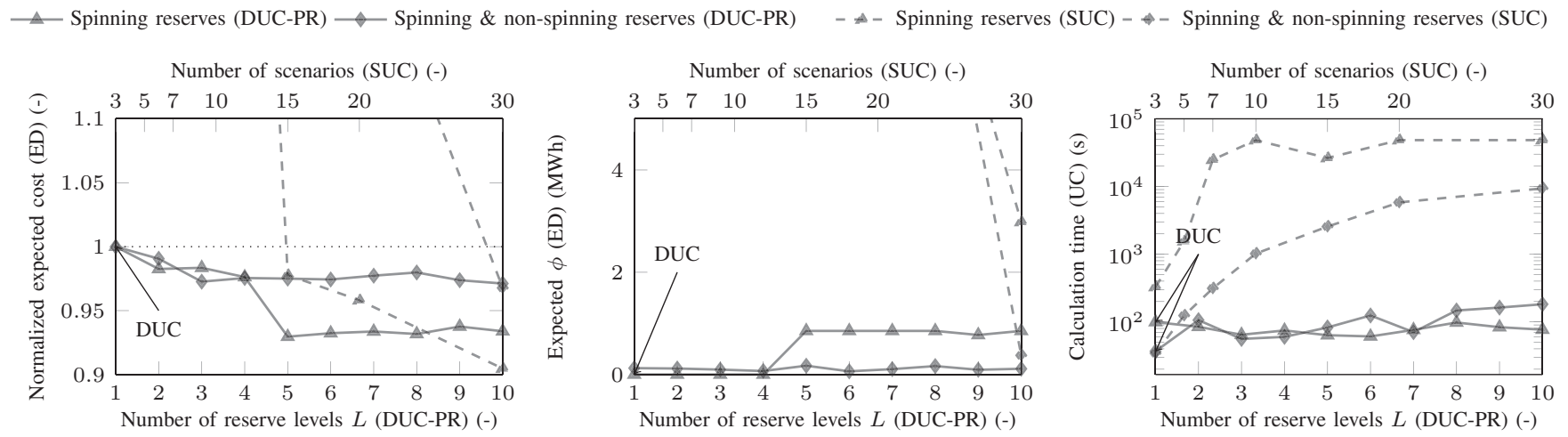

Fig. 2. Increasing the number of reserve levels typically leads to reduced operational costs (left), but increases the expected load shedding volume (center) and the calculation time (right). The operational cost is shown relative to the cost as obtained from the equivalent DUC model. The markers indicate the (un)availability of non-spinning reserves, the solid lines correspond to the results obtained with a DUC-PR model w.r.t. the number of reserve levels $L$ (bottom axis) and the dashed lines visualize the equivalent SUC results as a function of the number of scenarios considered (top axis).

The effect of the number of reserve levels $L$ on the expected operational cost, reliability and calculation time of the DUCPR schedule is illustrated in Fig. 2. The expected operational cost (Fig. 2, left) is normalized with respect to the operational cost as obtained from the corresponding DUC model. In the same figure, the performance of the equivalent SUC model as a function of the number of scenarios considered during the UC optimization is visualized.

Discretizing the reserve requirement and accounting for the activation costs of the scheduled reserves results in a significant drop in operational costs if one considers sufficient reserve levels (Fig. 2). Two effects are at play. First, the DUC-PR model will 'relax' reserve constraints by scheduling load shedding as a reserve provider during the UC phase (Fig. $3 \mathrm{~b}$ and $3 \mathrm{e}$ ). Although this results in some load shedding during dispatch (Fig. 2, center), the expected operational cost is significantly lower (Fig. 2, left). This effect is the strongest when one does not consider non-spinning reserves. Spinning reserves are typically provided by larger units, which makes following the upward reserve requirement exactly more difficult. To provide the last few MW's of upward reserve capacity, a new unit may have to be committed, which may result in a higher expected operational cost than curtailing some load. In other words, the allocation and expected activation cost of the last MW's of reserves is higher than the expected cost of a limited volume of load shedding. Non-spinning reserves are smaller in size and have lower allocation and expected activation costs if scheduled in a reserve level with a low activation probability. Hence, less load shedding is scheduled as a reserve provider in these cases. Second, the consideration of the activation costs allows scheduling an optimal mix of technologies to provide upward and downward flexibility. For example, when non-spinning and spinning reserves are available, the operational cost keeps decreasing while the load shedding volume remains constant. For this particular day, the second effect has a less pronounced impact on the operational cost (Fig. 2), but does affect the scheduled flexibility (Fig. 3). Note that the decrease in operational costs levels off above a certain number of reserve levels L. As of that point, more detailed representations of the distribution of the forecast error do not lead to further improvements in the UC schedule, but do increase the complexity of the problem, as illustrated by the moderate increase in calculation time (Fig. 2, right). Solving a DUC model takes 30 to 90 seconds. This calculation time increases to 80 to 190 seconds (DUC-PR, $L=10$ ). When non-spinning reserves are available, the complexity of the problem and the calculation time typically increase. The higher calculation times associated with the DUC-PR problems may be linked to the size of problem. The DUC problem contains approx. 240,000 equations and 170,000 variables, of which 55,000 are binary variables. The equivalent DUC-PR problem, considering 10 reserve levels, contains 462,000 equations and 752,000 variables, of which 191,000 are binary variables. Note however that the increase in calculation time with the number of reserve levels is moderate compared to the evolution of the calculation time of solving the extensive form of the SUC problem with the number of scenarios considered (Fig. 2, right).

The presented analysis is analogous to a solution stability study in stochastic programming [2], [9], [32]. If one takes too few scenarios into account, the SUC model will underestimate the required reserves, which results in high load shedding volumes (Fig. 2, center) and high operational costs (Fig. 2, left) during dispatch. In this particular case, 30 scenarios are required to obtain a so-called stable solution [2], [9]. Note that the evolution of the expected load-shedding volumes is opposite to that in the DUC-PR solutions. Starting from a UC schedule in which load shedding is not expected to occur, load shedding is gradually scheduled as a flexibility option with increasing granularity in the reserve levels in the DUCPR model. On the contrary, the SUC formulation starts from a UC schedule with high volumes of expected load shedding, reducing this volume as more detailed representations of the uncertain wind power forecast are considered (i.e. a higher number of scenarios). When non-spinning reserves are available, the complexity of the problem increases, but the calculation time decreases drastically. The quality of the resulting UC schedule is heavily dependent on the number of scenarios. Considering 30 scenarios, the SUC schedule outperforms the DUC-PR solution (Fig. 2, left). The calculation time required to solve the extensive form of the SUC problem however also strongly increases with the number of scenarios 


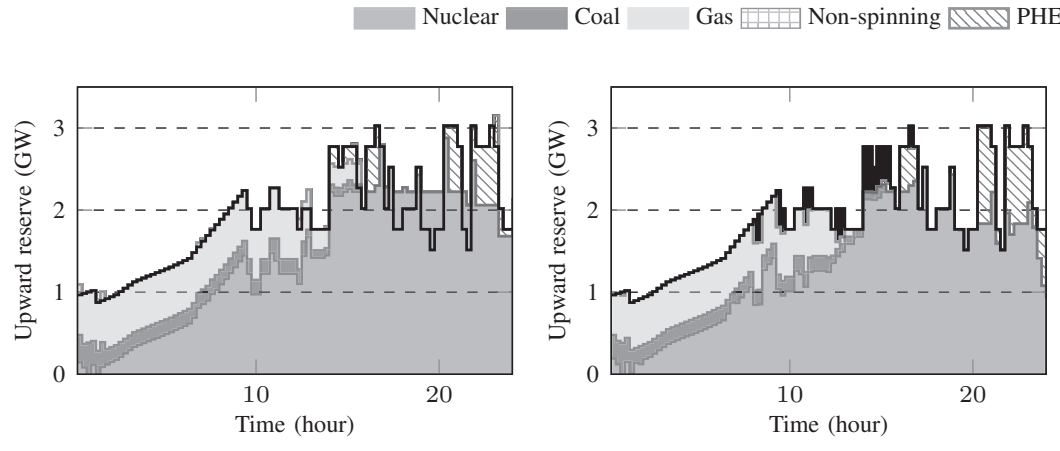

(a) DUC - Spinning reserves

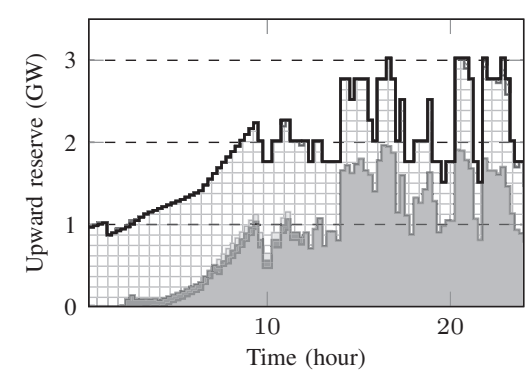

(d) DUC - Spinning \& non-spin. reserves (b) DUC-PR - Spinning reserves

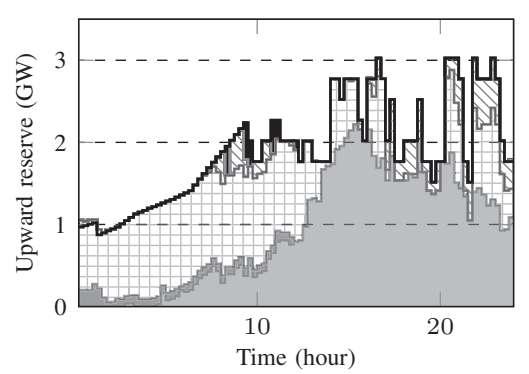

(e) DUC-PR - Spinning \& non-spin. reserves

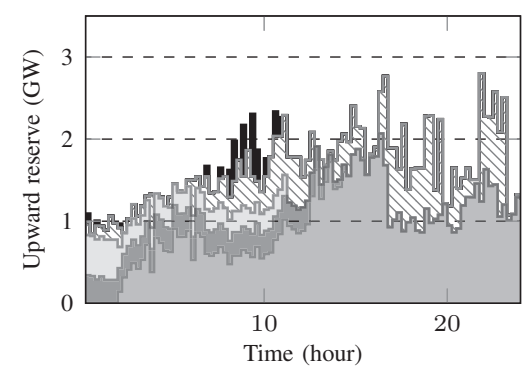

(c) SUC - Spinning reserves

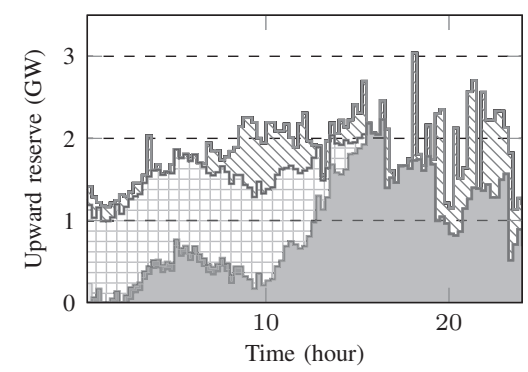

(f) SUC - Spinning \& non-spin. reserves

Fig. 3. The scheduled upward reserve capacity per technology and per UC formulation. In the top row, only spinning and PHES-based reserves are allowed. In the bottom row, non-spinning reserves are available. The solid black line indicates the total upward reserve requirement. The DUC-PR formulation employs five reserve levels $(L=5)$. The SUC model considers 30 scenarios.

considered and rises from approx. 750 seconds to 5,800 (5 scenarios) to 96,000 seconds (30 scenarios) (Fig. 2, right). The SUC problem size increases from approx. 1,000,000 equations and 642,000 variables, of which 245,000 binary variables, (5 scenarios) to $26,607,000$ equations and $14,635,000$ variables, of which $5,357,000$ are binary variables (30 scenarios). Note that decomposition techniques and parallelization - not explored in the context of this paper - may strongly reduce the computational cost of solving the SUC problems (see e.g. [8]).

Figure 3 illustrates the scheduled upward flexibility as obtained from a DUC, DUC-PR and SUC model for this particular day. The DUC-PR model contains five reserve levels. We will focus on the scheduled upward flexibility because of the large impact of possible load shedding. However, a similar analysis could be conducted for downward flexibility. Several effects are visualized in Fig. 3. First, the DUCPR model actively schedules load shedding $(\phi)$ as upward flexibility, lowering the effective upward reserve requirement. As a result, fewer units have to be committed (Fig. 3a-3b and $3 \mathrm{~d}-3 \mathrm{e})$. When non-spinning reserves are available, more costeffective low-activation-probability flexibility is available to meet the reserve requirement. Significantly less load shedding is scheduled (Fig. 3d-3e). Second, as non-spinning reserves by assumption do not present any allocation costs and activation costs are not considered in the DUC formulation, all units that can provide non-spinning reserves are scheduled by the DUC model (Fig. 3d). The DUC-PR model typically schedules less non-spinning reserves and more spinning reserves (nuclear) due to the high expected activation cost of these non-spinning reserves (Fig. 3e). Third, less curtailment is scheduled when non-spinning reserves are available. Fourth, both the DUC and
DUC-PR schedules remain conservative compared to the SUC solution. Less capacity is scheduled by the SUC model, while similar load shedding volumes are attained during dispatch (Fig. 2, center). Especially the potential regulation services of the PHES system remain underestimated in the DUCPR formulation due to the conservative worst-case feasibility constraints [26]. For a detailed discussion on the coupling between PHES-based reserves and UC optimization, the reader is referred to [26].

\section{B. Performance of the DUC-PR formulation}

To evaluate the performance of the DUC-PR formulation quantitatively, four representative weeks were selected based on the residual demand ${ }^{5}$. The week with the residual demand closest to the average weekly demand for electrical energy (week 30), the week with the lowest residual energy demand (week 52), the week with the highest residual energy demand (week 9) and the week with the highest variability in the residual load profile (week 39) were selected. For these weeks, expected operational costs, curtailment of RES-based generation and loss of load volumes were calculated based on the SUC model considering 30 scenarios, as well as with the proposed DUC-PR model considering 5 reserve levels and the equivalent DUC model. For the expected operational cost $\mathrm{E}[\mathrm{TOC}]$, we calculate the $95 \%$ confidence interval $[E[T O C]-$ $\Delta, E[T O C]+\Delta]$ via the minimum variance method [33], as recommended in [34].

The SUC results will serve as a benchmark, although solution stability may not be reached in all instances [2]. Especially

\footnotetext{
${ }^{5}$ This residual demand is calculated as the difference between the historical demand time series and the rescaled historical wind power time series.
} 
when non-spinning reserves may not be scheduled, the SUC model relies on the PHES system to offer regulation services. By definition, in absence of so-called bundle constraints, linking the output or energy storage levels in the different scenarios, the PHES schedule will be optimized toward the considered scenarios [21]. Other possible realizations of the uncertain RES-based generation may contain events that are not captured by the scenario set considered during the UC optimization [21]. In this particular case, the SUC model overestimates the available flexibility of the PHES system, which results in high volumes of load shedding, hence high operational costs and wide confidence intervals, in some instances (Table I). However, due to the computational burden of solving the extensive form of the SUC problem, we will limit the number of scenarios considered in the SUC problems to 30 .

During week 30 (average residual demand), the performance of the DUC-PR and DUC models is comparable. Curtailment does not occur. The SUC model is outperformed by the DUC and DUC-PR formulations. The UC schedule obtained with the SUC model is inadequate to meet the demand without load shedding in some scenarios, resulting in high expected load shedding volumes and, consequently, increased expected operational costs. In addition, load shedding in certain extreme scenarios increases the variance in the operational cost per scenario, resulting in a wide confidence interval $(13.9+/-$ $0.36 \mathrm{M} € /$ week and $13.3+/-0.25 \mathrm{M} € /$ week respectively).

Similar effects are observed in week 9 (maximum residual demand). The DUC-PR formulation however realizes operational cost savings of approx. $0.1 \mathrm{M} €$ or $0.4 \%$ per week (compared to the DUC results) to $0.5 \mathrm{M} €$ or $1.8 \%$ per week (compared to the SUC model). Load shedding volumes are similar in the DUC and DUC-PR results, and significantly lower than in the SUC results. Consequently, the $95 \%$ confidence interval on the expected operational cost of the SUC schedule is significantly wider than the same confidence interval on the DUC and DUC-PR results. Curtailment does not occur. When considering non-spinning reserves, the performance of the DUC and DUC-PR models is similar and 0.1 M€ per week worse than that of the SUC formulation.

During periods of low residual demand (week 52), curtailment and downward flexibility become decisive for the performance of the UC models. The DUC-PR model explicitly considers the possible cost savings of downward flexibility, which results in operational cost savings of $0.4 \mathrm{M} €$ per week $(14 \%)$ to $0.1 \mathrm{M} €$ per week $(4.2 \%)$. The difference in operational cost and curtailment is typically smaller when non-spinning reserves may be scheduled. As the DUC model does not see the cost of activation of these units and their allocation costs are by assumption set to zero, all of them are scheduled throughout the week. Although this is sub-optimal, the resulting UC schedule is highly flexible, resulting in an operational cost that approximates that of the DUC-PR schedule. Convergence issues result in high load shedding volumes, thus high expected operational costs and wide confidence intervals, in the SUC solutions.

If the residual demand profile is strongly variable (week 39), the DUC-PR formulation outperforms the DUC formulation
TABLE I

COMPARISON OF THE DUC, DUC-PR AND SUC RESUlTS AFTER MONTE CARLO ECONOMIC DISPATCH ON 500 WIND POWER SCENARIOS PER DAY FOR FOUR REPRESENTATIVE WEEKS OF THE YEAR. TOC IS THE TOTAL OPERATIONAL COST, WITH $\Delta$ A METRIC FOR THE WIDTH OF THE $95 \%$ CONFIDENCE INTERVAL. TOC* IS THE TOC EXCLUDING THE COST ASSOCIATED WITH LOAD NOT SERVED. WUF IS THE WIND UTILIZATION FACTOR, DEFINED AS THE PERCENTAGE OF AVAILABLE WIND ENERGY THAT IS ABSORBED IN THE SYSTEM. WS IS THE WIND SHARE, I.E. THE EFFECTIVE SHARE OF WIND ENERGY IN THE GENERATED ENERGY IN THIS WEEK, CORRECTED FOR CURTAILMENT. $\phi$ IS THE LOAD SHEDDING VOLUME. All VALUES ARE EXPECTED VALUES (E[]).

\begin{tabular}{lc|ccc|ccc}
\hline \hline & & \multicolumn{3}{|c|}{ Spin. } & \multicolumn{3}{c}{ Spin. \& non-spin. } \\
& & SUC & DUC-PR & DUC & SUC & DUC-PR & DUC \\
\hline \hline E[TOC] & $(\mathrm{M} €)$ & 13.9 & 13.7 & 13.8 & 13.3 & 13.3 & 13.2 \\
$\Delta$ & $(\mathrm{M} €)$ & 0.36 & 0.08 & 0.08 & 0.25 & 0.10 & 0.10 \\
$\left.\mathrm{E}_{\mathrm{TOC}}^{*}\right]$ & $(\mathrm{M} €)$ & 13.4 & 13.7 & 13.8 & 13.0 & 13.3 & 13.2 \\
$\mathrm{E}[\mathrm{WUF}]$ & $(\%)$ & 100 & 100 & 100 & 100 & 100 & 100 \\
$\mathrm{E}[\phi]$ & $(\mathrm{MWh})$ & 55.0 & 0 & 0 & 36.0 & 1.8 & 0.7 \\
$\mathrm{E}[\mathrm{WS}]$ & $(\%)$ & 10.6 & 10.6 & 10.6 & 10.6 & 10.6 & 10.6 \\
\hline \hline
\end{tabular}

(a) Average residual demand (week 30).

\begin{tabular}{lc|ccc|ccc}
\hline \hline & & \multicolumn{3}{|c|}{ Spin. } & \multicolumn{3}{c}{ Spin. \& non-spin. } \\
& & SUC & DUC-PR & DUC & SUC & DUC-PR & DUC \\
\hline \hline E[TOC] & $(\mathrm{M} €)$ & 4.2 & 2.9 & 3.3 & 3.1 & 2.3 & 2.4 \\
$\Delta$ & $(\mathrm{M} €)$ & 0.84 & 0.04 & 0.01 & 0.56 & 0.05 & 0.03 \\
$\mathrm{E}\left[\mathrm{TOC}^{*}\right]$ & $(\mathrm{M} €)$ & 2.6 & 2.9 & 3.3 & 2.2 & 2.3 & 2.4 \\
$\mathrm{E}[\mathrm{WUF}]$ & $(\%)$ & 92.4 & 87.5 & 86.7 & 94.5 & 91.6 & 92.4 \\
$\mathrm{E}[\phi]$ & $(\mathrm{MWh})$ & 146.6 & 3.0 & 0 & 82.6 & 4.0 & 0.1 \\
$\mathrm{E}[\mathrm{WS}]$ & $(\%)$ & 79.7 & 75.4 & 74.8 & 81.8 & 79.0 & 79.7 \\
\hline \hline
\end{tabular}

(b) Minimum residual demand (week 52).

\begin{tabular}{lc|ccc|ccc}
\hline \hline & & \multicolumn{3}{|c|}{ Spin. } & \multicolumn{3}{c}{ Spin. \& non-spin. } \\
& & SUC & DUC-PR & DUC & SUC & DUC-PR & DUC \\
\hline \hline E[TOC] & $(\mathrm{M} €)$ & 28.9 & 28.4 & 28.5 & 27.4 & 27.5 & 27.5 \\
$\Delta$ & $(\mathrm{M} €)$ & 0.84 & 0.16 & 0.16 & 0.37 & 0.20 & 0.19 \\
$\mathrm{E}\left[\mathrm{TOC}^{*}\right]$ & $(\mathrm{M} €)$ & 27.9 & 28.4 & 28.5 & 27.1 & 27.5 & 27.5 \\
$\mathrm{E}[\mathrm{WUF}]$ & $(\%)$ & 100 & 100 & 100 & 100 & 100 & 100 \\
$\mathrm{E}[\phi]$ & $(\mathrm{MWh})$ & 109.5 & 0 & 0 & 31.5 & 3.0 & 1.8 \\
$\mathrm{E}[\mathrm{WS}]$ & $(\%)$ & 13.5 & 13.5 & 13.5 & 13.5 & 13.5 & 13.5 \\
\hline \hline
\end{tabular}

(c) Maximum residual demand (week 9).

\begin{tabular}{lc|ccc|ccc}
\hline \hline & & \multicolumn{3}{|c|}{ Spin. } & \multicolumn{3}{c}{ Spin. \& non-spin. } \\
& & SUC & DUC-PR & DUC & SUC & DUC-PR & DUC \\
\hline \hline $\mathrm{E}[\mathrm{TOC}]$ & $(\mathrm{M} €)$ & 8.5 & 8.4 & 8.8 & 6.3 & 6.7 & 6.9 \\
$\Delta$ & $(\mathrm{M} €)$ & 0.99 & 0.07 & 0.06 & 0.68 & 0.11 & 0.10 \\
$\mathrm{E}\left[\mathrm{TOC}{ }^{*}\right]$ & $(\mathrm{M} €)$ & 7.4 & 8.4 & 8.8 & 5.6 & 6.7 & 6.9 \\
$\mathrm{E}[\mathrm{WUF}]$ & $(\%)$ & 96.4 & 93.8 & 93.5 & 97.9 & 96.0 & 96.0 \\
$\mathrm{E}[\phi]$ & $(\mathrm{MWh})$ & 115.5 & 0.2 & 0 & 73.2 & 0.7 & 0.2 \\
$\mathrm{E}[\mathrm{WS}]$ & $(\%)$ & 50.4 & 49.1 & 48.9 & 51.2 & 50.2 & 50.3 \\
\hline \hline
\end{tabular}

(d) Maximum variability in residual demand (week 39).

by 0.2 to $0.4 \mathrm{M} € /$ week (3.0 to $4.7 \%$ respectively). This is the result of scheduling load shedding as upward reserves, which allows covering the upward reserve requirement with less online capacity and to a lesser extent, the scheduling of a more cost-efficient mix of technologies to meet the reserve requirement. The SUC model outperforms the DUC-PR formulation considering non-spinning reserves, despite a higher volume load shedding, which results in wide confidence intervals, in the SUC solutions. The resulting WUF is significantly higher. 
TABLE II

MEDIAN, $75^{\text {th }}$ AND $95^{\text {th }}$ PERCENTILE OF THE CALCULATION TIME (PER UC PROBLEM) FOR THE SUC, DUC AND DUC-PR MODEL.

\begin{tabular}{cc|ccc|ccc}
\hline \hline & & \multicolumn{3}{|c|}{ Spin. } & \multicolumn{3}{c}{ Spin. \& non-spin } \\
& & SUC & DUC-PR & DUC & SUC & DUC-PR & DUC \\
\hline \hline $\mathrm{P}_{50}$ & $(\mathrm{~s})$ & 19,897 & 201 & 138 & 24,833 & 281 & 125 \\
$\mathrm{P}_{75}$ & $(\mathrm{~s})$ & 46,748 & 267 & 191 & 57,256 & 365 & 213 \\
$\mathrm{P}_{95}$ & $(\mathrm{~s})$ & $+96,000$ & 404 & 342 & $+96,000$ & 534 & 395 \\
\hline \hline
\end{tabular}

\section{Computational performance}

Table II compares the calculation times (median, $75^{\text {th }}$ and $95^{\text {th }}$ percentile) for the DUC, DUC-PR and SUC problems. Solving a DUC problem takes approx. two minutes (median values). Introducing non-spinning reserves does not significantly impact the calculation time of the DUC problems. As discussed above, the DUC formulation does not 'see' the complex trade-off between activation and allocation costs, resulting in fast models, but sub-optimal UC schedules. The spread on the calculation time is limited: $95 \%$ of all simulations yield a solution in less than 6 minutes.

Solving a DUC-PR problem takes 3.3 to 4.8 minutes (median values). Considering non-spinning reserves considerably increases the calculation time. $95 \%$ of all simulations terminate within 6.7 minutes (without non-spinning reserves) and 8.9 minutes (with non-spinning reserves). However, this represents a significant improvement in calculation time compared to solving the extensive form of the SUC problem, which requires approx. 5.5 to 6.9 hours (median values). Recall that we did not consider decomposition techniques and parallelization, which may strongly reduce the computational cost of solving the SUC problems [8].

\section{CONCLUSION}

Although the accuracy of RES-based electricity generation forecasts is improving, power system operators need to procure up- or downward regulation capacity under the form of dispatchable generation or load to overcome inevitable deviations from the forecasted RES-based generation profile. Stochastic unit commitment (SUC) models, with a direct representation of the uncertainty via a set of scenarios in the UC model, lead to an optimal trade-off between reliability and expected operational system cost. However, these SUC models may be computationally costly to solve and the quality of the resulting UC schedule is highly dependent on the scenarios considered. In this paper we therefore develop a novel set of probabilistic reserve requirements. By defining distinct reserve levels, each with a probability of activation, one can account for the allocation and expected activation costs in a deterministic UC model, without the need of a scenario-based representation of the uncertain RES-based generation. This improves the performance of the resulting UC schedule considerably, as shown in Section III-B. The reason behind these operational cost reductions is twofold. First, the model is able to account for possible operational cost savings resulting from the activation of downward reserves. Although excess RES-based generation can be curtailed, not absorbing unexpected increases in RESbased generation may be sub-optimal. Second, activation probabilities allow providing upward reserves cost-effectively by a mix of (1) cheap, frequently activated running power plants (spinning reserves), (2) expensive, but rarely activated offline power plants (non-spinning reserves), (3) energy storage-based flexibility, (4) load shedding and (5) scheduled curtailment of forecasted RES-based generation. Without the inclusion of activation probabilities, this trade-off was not possible in a DUC model. This allows us to approximate the operational cost of the stable solution of a SUC model in calculation times similar to that of a deterministic formulation.

The presented DUC-PR formulation can be used to assess the impact of uncertainty on reasonably large low-carbon electric power systems where SUC models could become computationally intractable. Likewise, independent system operators (ISO) could use this model to optimize their UC decisions taking into account the uncertainty in their system.

This work may be strengthened in the following ways. First, considering multiple sources of uncertainty and studying their interaction may increase the added value of this work. Second, expanding the presented model to consider transmission constraints would allow for a number of interesting applications. For example, employing this model on multiple interconnected areas would allow studying how pooling of reserves across areas, as well as the interaction of uncertainty in different areas, affects reserve allocation and activation costs. Third, the dependency of the obtained results on the assumed underlying distribution could be studied, as the approach above requires knowledge of the probability of activation of each power plant offering reserves at each time step. Wrong estimates of these probabilities may have a significant impact on the performance of the obtained UC schedule. Fourth, better discretization schemes may exist and merit further research, but are out of the scope of the present paper.

\section{ACKNOWLEDGMENT}

The computational resources and services used in this work were provided by the VSC (Flemish Supercomputer Center), funded by the Research Foundation - Flanders (FWO) and the Flemish Government - department EWI. K. Bruninx is a postdoctoral research fellow of the Research Foundation - Flanders (FWO) and VITO, the Flemish Institute for Technological Research.

\section{REFERENCES}

[1] M. A. Ortega-Vazquez and D. S. Kirschen, "Optimizing the Spinning Reserve Requirements Using a Cost/Benefit Analysis," IEEE Trans. Power Syst., vol. 22, no. 1, pp. 24-33, 2007.

[2] K. Bruninx, K. Van Den Bergh, E. Delarue, and W. D'haeseleer, "Optimization and allocation of spinning reserves in a low carbon framework," IEEE Trans. Power Syst., vol. 31, no. 2, pp. 872-882, 2016.

[3] S. Cerisola, A. Baíllo, J. M. Fernández-López, A. Ramos, and R. Gollmer, "Stochastic Power Generation Unit Commitment in Electricity Markets: A Novel Formulation and a Comparison of Solution Methods," Operations Research, vol. 57, no. 1, pp. 32-46, 2009.

[4] S. M. Ryan, R. J.-B. Wets, D. L. Woodruff, C. Silva-Monroy, and J.-P. Watson, "Toward scalable, parallel progressive hedging for stochastic unit commitment," in IEEE PES GM. Vancouver, BC, Canada: IEEE, July 21-25, 2013, pp. 1-5.

[5] S. Takriti, J. Birge, and E. Long, "A stochastic model for the unit commitment problem," IEEE Trans. Power Syst., vol. 11, no. 3, pp. 1497-1508, 1996. 
[6] R. Gollmer, M. P. Nowak, W. Römisch, and R. Schultz, "Unit commitment in power generation - a basic model and some extensions," Annals of Operations Research, vol. 96, no. 1, pp. 167-189, 2000.

[7] A. Papavasiliou, S. S. Oren, and B. Rountree, "Applying High Performance Computing to Transmission-Constrained Stochastic Unit Commitment for Renewable Energy Integration," IEEE Trans. Power Syst., vol. 30, no. 3, pp. 1109-1120, May 2015.

[8] K. Cheung, D. Gade, C. Silva-Monroy, S. M. Ryan, J.-P. Watson, R. J.-B. Wets, and D. L. Woodruff, "Toward scalable stochastic unit commitment - part 2: solver configuration and performance assessment," Energy Systems, vol. 6, no. 3, pp. 417-438, 2015.

[9] K. Bruninx and E. Delarue, "Scenario Reduction Techniques and Solution Stability for Stochastic Unit Commitment Problems," in ENERGYCON 2016. Leuven, Belgium: IEEE, April 4-8, 2016, pp. 1-7.

[10] J. Morales and S. Pineda, "Scenario reduction for futures market trading in electricity markets," IEEE Trans. Power Syst., vol. 24, no. 2, pp. 878888, 2009.

[11] P. Ruiz, C. Philbrick, and P. Sauer, "Modeling Approaches for Computational Cost Reduction in Stochastic Unit Commitment Formulations," IEEE Trans. Power Syst., vol. 25, no. 1, pp. 588-589, 2010.

[12] Y. Dvorkin, Y. Wang, H. Pandžić, and D. Kirschen, "Comparison of scenario reduction techniques for the stochastic unit commitment," in IEEE PES GM. New Harbor, MD, USA: IEEE, July 27-31, 2014, pp. $1-5$.

[13] Y. Wang, Q. Xia, and C. Kang, "Unit Commitment With Volatile Node Injections by Using Interval Optimization," IEEE Trans. Power Syst., vol. 26 , no. 3 , pp. $1705-1713,2011$.

[14] K. Bruninx and E. Delarue, "A Statistical Description of the Error on Wind Power Forecasts for Probabilistic Reserve Sizing," IEEE Trans. Sustain. Energy, vol. 5, no. 3, pp. 995-1002, 2014.

[15] K. De Vos and J. Driesen, "Dynamic operating reserve strategies for wind power integration," IET Renew. Power Gen., vol. 8, no. 6, pp. 598-610, 2014.

[16] D. Bertsimas, E. Litvinov, X. A. Sun, J. Zhao, and T. Zheng, "Adaptive Robust Optimization for the Security Constrained Unit Commitment Problem," IEEE Trans. Power Syst., vol. 28, no. 1, pp. 52-63, 2013.

[17] H. Pandžić, Y. Dvorkin, Y. Wang, T. Qiu, and D. S. Kirschen, "Effect of time resolution on unit commitment decisions in systems with high wind penetration," in IEEE PES GM. National Harbor, MD, USA: IEEE, July 27-31, 2014, pp. 1-5.

[18] U. A. Ozturk, M. Mazumdar, and B. Norman, "A solution to the stochastic unit commitment problem using chance constrained programming," IEEE Trans. Power Syst., vol. 19, no. 3, pp. 1589-1598, 2004.

[19] G. Yongpei and J. Wang, "Uncertainty Sets for Robust Unit Commitment," IEEE Trans. Power Syst., vol. 29, no. 3, pp. 1439-1440, 2014.

[20] D. Bertsimas and M. Sim, "The price of robustness," Operations research, vol. 52, no. 1, pp. 35-53, 2004.

[21] K. Bruninx, "Improved modeling of unit commitment decisions under uncertainty," PhD dissertation, KU Leuven, 2016. [Online]. Available: http://www.mech.kuleuven.be/en/tme/research/ energy_environment/Pdf/doctoraat-kenneth-bruninx.pdf

[22] D. Pozo and J. Contreras, "A Chance-Constrained Unit Commitment With an n-K Security Criterion and Significant Wind Generation," IEEE Trans. Power Syst., vol. 28, no. 3, pp. 2842-2851, 2013.

[23] J. F. Restrepo and F. D. Galiana, "Assessing the yearly impact of wind power through a new hybrid deterministic/stochastic unit commitment," IEEE Trans. Power Syst., vol. 26, no. 1, pp. 401-410, 2011.

[24] J. Xiao, B. M. S. Hodge, J. F. Pekny, and G. V. Reklaitis, "Operating reserve policies with high wind power penetration," Comp. Chem. Eng., vol. 35, no. 9, pp. 1876-1885, 2011.

[25] P. Pinson, H. Madsen, H. A. Nielsen, G. Papaefthymiou, and B. Klöckl, "From probabilistic forecasts to statistical scenarios of short-term wind power production," Wind Energy, vol. 12, no. 1, pp. 51-62, 2009.

[26] K. Bruninx, Y. Dvorkin, E. Delarue, H. Pandzic, W. Dhaeseleer, and D. S. Kirschen, "Coupling Pumped Hydro Energy Storage With Unit Commitment," IEEE Trans. Sustain. Energy, vol. 7, no. 2, pp. 786-796, 2016.

[27] E. Lannoye, D. Flynn, and M. O’Mally, "Transmission, Variable Generation, and Power System Flexibility," IEEE Trans. Power Syst., vol. 30 no. 1, pp. 1-10, 2015.

[28] K. Van den Bergh, D. Couckuyt, E. Delarue, and W. D'haeseleer, "Redispatching in an interconnected electricity system with high renewables penetration," Electric Power System Research, vol. 127, no. 10, pp. 64 $72,2015$.

[29] A. Papavasiliou, S. S. Oren, and R. P. O’Neill, "Reserve requirements for wind power integration: A scenario-based stochastic programming framework," IEEE Trans. Power Syst., vol. 26, no. 4, pp. 2197-2206, 2011.

[30] Elia Group, "Elia Grid Data," 2013. [Online]. Available: www.elia.be

[31] N. Troy, D. Flynn, and M. O'Malley, "The importance of sub-hourly modeling with a high penetration of wind generation," in IEEE PES GM. San Diego, CA, USA: IEEE, July 22-26, 2012, pp. 1-6.

[32] M. Kaut and S. W. Wallace, "Evaluation of scenario-generation methods for stochastic programming," Pacific Journal of Optimization, vol. 3 , no. 2, pp. 257-271, 2007.

[33] G. Hahn and S. Shapiro, Statistical Models in Engineering. New York, NY, USA: Wiley, 1967.

[34] H. Pandžić, Y. Dvorkin, T. Qiu, Y. Wang, and D. S. Kirschen, "Toward Cost-Efficient and Reliable Unit Commitment Under Uncertainty," IEEE Trans. Power Syst., vol. 31, no. 2, pp. 970-982, 2015.

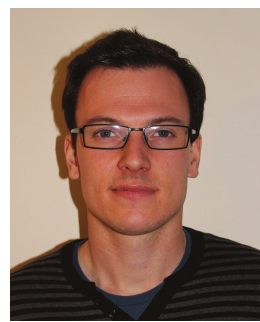

Kenneth Bruninx (S'15) received the M.Sc. degree in energy engineering in 2011 and the Ph.D. degree in mechanical engineering in 2016, both from the Univeristy of Leuven (KU Leuven), Belgium.

Currently, he is working as a post-doctoral research fellow of the Research Foundation - Flanders (FWO) at (i) the University of Leuven Energy Institute, TME branch (energy conversion), (ii) VITO (the Flemish Institute for Technological Research) and (iii) EnergyVille, a joint venture of KU Leuven, VITO and IMEC.

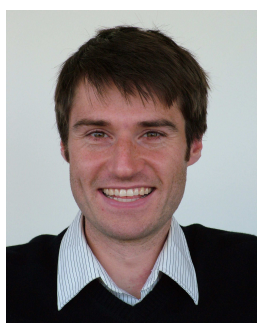

Erik Delarue (M'15) received the M.Sc. degree in mechanical engineering in 2005 and the Ph.D degree in mechanical engineering in 2009, both from the University of Leuven (KU Leuven), Belgium.

Currently, he is an Assistant Professor at the University of Leuven Energy Institute, TME branch (energy conversion). 